UTTG-02-98

hep-th/9803048

March 5, 1998

\title{
Matrix Theory on ALE Spaces and Wrapped Membranes
}

\author{
David Berenstein and Richard Corrado * \\ Theory Group, Department of Physics \\ University of Texas at Austin \\ Austin TX 78712 USA \\ Email: david@zippy.ph.utexas.edu \\ Email: rcorrado@zippy.ph.utexas.edu
}

\begin{abstract}
We study the properties of wrapped membranes in matrix theory on ALE spaces. We show that the only BPS bound states of wrapped membranes that can form are roots of the $A-D-E$ group. We determine a bound on the energy of a bound state and find the correct dependence on the blow-up parameters and longitudinal momentum expected from M-Theory. For the $A_{n-1}$ series, we construct explicit classical solutions for the wrapped membrane bound states. These states have a very rich structure and have a natural interpretation in terms of noncommutative geometry. In the $A_{1}$ case, we examine the spectrum of excitations around the wrapped membrane solution and provide an explicit calculation of their energies. The results agree exactly with supergravity calculations.
\end{abstract}

\footnotetext{
* Research supported in part by the Robert A. Welch Foundation and NSF Grant PHY 9511632.
} 


\section{Introduction}

The M(atrix)-Theory [1] proposal for a non-perturbative description of M-Theory has been demonstrated to properly capture M-Theory physics in a variety of settings (see [2-4] for recent reviews). However, several important areas where our understanding of matrix theory is incomplete still remain. For example, the fact that matrix theory captures the physics of linearized supergravity is fairly well understood [5]. The reasons for its apparent failure to capture supergravity results in other situations [6-9] seem to be subtleties in our understanding of the discrete light-cone quantization (DLCQ) of M-Theory, namely that the low-energy description of DLCQ M-Theory is not quite supergravity [2,10,3].

Another area which requires further study is the compactification of matrix theory, in particular on curved manifolds. One promising case is that of "compactification" on ALE spaces, which has been conjectured to be described by the theory of D0-brane partons moving on the ALE space $[11,6,12,13]$.

In this paper we continue the study of matrix theory on ALE spaces. In Section 2, we give a very brief review of ALE matrix theory. Our emphasis will be to further examine the characteristics of the description of membranes which are wrapped around homology 2-cycles as recently described in [14-16]. In Section 3, we focus on the existence of wrapped membrane BPS bound states in the wrapped membrane matrix model presented in [16]. We demonstrate that such bound states must be roots under the $A-D-E$ group. We then derive a bound on the energy of such a bound state. For the case of the $A_{n-1}$ series, we explicitly construct the bound states for all roots. In Section 4, we briefly discuss how the membrane solution fits into the framework of noncommutative geometry and thereby satisfies the properties of spherical membranes $[17,18]$. In Section 5, we discuss the spectrum of excitations of the wrapped membrane and calculate the energies of excitations around the $A_{1}$ solution, finding agreement with supergravity expectations.

\section{A Brief Review of ALE Matrix Theory}

A matrix description of M-Theory on an ALE space must possess several crucial ingredients if it is to be considered both correct and useful. A candidate formalism for such a description has been provided by the worldvolume effective theories describing D0-branes moving on an ALE space [19-21]. Let us briefly review the construction of these models and examine the spacetime features of M-Theory that they capture.

The ALE matrix models are given by the dimensional reduction to quantum mechanics of the six-dimensional gauged supersymmetric sigma models appearing in the hyperkähler 


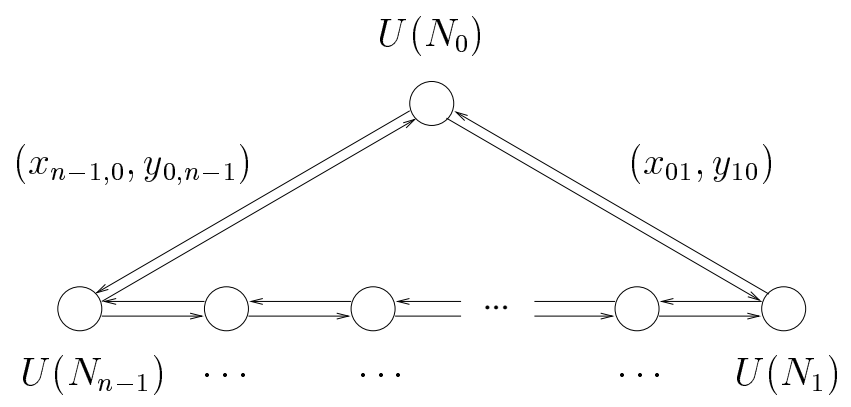

Figure 1: The $A_{n-1}$ quiver diagram, with representative $U\left(N_{i}\right)$ gauge groups at the vertices and hypermultiplets, $\left(x_{i, i+1}, y_{i+1, i}\right)$, on the edges.

quotient construction $[22,23]$ of the ALE space. The field content is summarized by a quiver diagram, which is based on the $A-D-E$ extended Dynkin diagram, such as the $A_{n-1}$ diagram shown in Figure 1. For each vertex of the diagram there is a gauge group $U\left(N_{i}\right)$, where $N_{i}=N k_{i}$, with $N$ the number of D0-branes and $k_{i}$ the Dynkin label of the vertex, as well as a vector multiplet, $\left(A_{i}, a_{i}\right)$ (in terms of $d=4, \mathcal{N}=1$ vector and chiral superfields), in the $\left(1, \ldots, \operatorname{ad}\left(U\left(N_{i}\right)\right), \ldots, 1\right)$ representation. For each edge of the diagram there is an associated hypermultiplet, $\left(x_{i, i+1}, y_{i+1, i}\right)$, in the fundamental-anti-fundamental representation, $\left(1, \ldots, 1, N_{i}, \bar{N}_{i+1}, 1, \ldots, 1\right)$, of the neighboring gauge groups.

The Lagrangian is the most general one compatible with the gauge symmetry and $d=6$, $\mathcal{N}=1$ SUSY. However, since we are in $d=1$ dimensions, the question of the mass dimension of the fields and coupling constants is an issue. We will choose string units, with $T_{A}=1$. All fields have mass dimension -1 and the coupling constant has dimension $-\frac{1}{2}$. This requires us to scale the hypermultiplets so that an inverse square of a coupling constant appears out in front of the terms in the Lagrangian that they appear in. We will choose this to be the "average" coupling constant, defined by

$$
\frac{1}{g^{2}}=\sum_{i} \frac{k_{i}}{g_{i}^{2}} .
$$

We also scale the $\mathrm{D}$ and F-terms by $2 / g^{2}$. In terms of $d=4, \mathcal{N}=1$ superfields, our 
Lagrangian is

$$
\begin{aligned}
\mathcal{L}=\sum_{i} & {\left[\left(\frac{1}{16 N_{i} g_{i}^{2}} \int d^{2} \theta W_{i}^{2}+\text { c.c. }\right)+\frac{1}{g_{i}^{2}} \int d^{4} \theta \bar{a}_{i} e^{A_{i}} a_{i} e^{-A_{i}}\right.} \\
+ & \frac{1}{g^{2}} \int d^{4} \theta\left(\bar{x}_{i, i+1} e^{A_{i}} x_{i, i+1} e^{-A_{i+1}}+\bar{y}_{i+1, i} e^{A_{i+1}} y_{i+1, i} e^{-A_{i}}\right) \\
+ & \left(\frac{1}{g^{2}} \int d^{2} \theta\left(y_{i+1, i} a_{i} x_{i, i+1}-x_{i-1, i} a_{i} y_{i, i-1}\right)+\text { c.c. }\right) \\
+ & \left.\frac{2}{g^{2}} \int d^{4} \theta d_{i} A_{i}+\left(\frac{2}{g^{2}} \int d^{2} \theta f_{i} a_{i}+\text { c.c. }\right)\right] .
\end{aligned}
$$

The coupling constant satisfies

$$
\frac{1}{g^{2}}=T_{D 0}
$$

Then it is clear that the $1 / g_{i}^{2}$ are the masses of the fractional D0-branes $[20,11,15]$ associated to each vertex.

The coefficients of the $\mathrm{D}$ and F-terms are constrained by the requirement that a supersymmetric ground state exists. This requires that

$$
\sum_{i} k_{i} f_{i}=\sum_{i} k_{i} d_{i}=0
$$

For $N_{i}=N k_{i}$, there are zero-energy ground states. Under the $S U(2)_{R}$ R-symmetry, the $\vec{\zeta}_{i}=\left(f_{i}, d_{i}\right)$ form triplets. In fact, according to the hyperkähler quotient construction, the $\vec{\zeta}_{i}$ are the blow-up parameters for the ALE space. With the normalization chosen in (2.2), the area of the $i^{\text {th }} \mathbb{P}^{1}$ is given by $4 \pi\left|\vec{\zeta}_{i}\right|^{2}$.

For simplicity, one typically chooses all the gauge couplings to be the same, but this is not required. As we will see later, these coupling constants have an interpretation as Wilson lines for the $A-D-E$ gauge group in the DLCQ M-Theory description.

Applying the matrix prescription [1], one is lead to conjecture that, in the limit $N \rightarrow \infty$, $g \rightarrow \infty$, the D0-branes described by (2.2) are the partons of the infinite-momentum frame description of M-Theory on the ALE space [11,6]. On the other hand, the finite $N$, finite $g$ matrix models would be conjectured to provide a description of the DLCQ of M-Theory [24] on the ALE space [8].

Several pieces of evidence support the M-Theory interpretation of these ALE matrix models. First, the models contain the geometry of the ALE space. For $N=1$, we have the standard $U(1)$ gauged supersymmetric sigma model. After taking the hyperkähler quotient [22], we recover the target space $\mathbb{R}^{5} \times \mathcal{M}_{\vec{\zeta}}$. Here we use $\mathcal{M}_{\vec{\zeta}}$ to denote the ALE space 
parameterized by the blowup parameters $\vec{\zeta}$. For larger $N$, the moduli space of the theory is

$$
\left(\mathbb{R}^{5} \times \mathcal{M}_{\vec{\zeta}}\right)^{N} / S_{N}
$$

so that the ALE geometry is recovered along the flat directions of the classical ground states of the system.

The models have the equivalent of $\mathcal{N}=1$ supersymmetry in six dimensions, or eight supercharges, which is the same amount of supersymmetry present in light-cone M-Theory on an ALE space. The gauge group $\prod_{i} U\left(N_{i}\right)$ contains an overall $U(1)$ factor which is a linear combination of the $U(1)$ s for each $U\left(N_{i}\right)$ factor. The vector associated to this $U(1)$ is decoupled from the rest of the dynamics. In the matrix model, these decoupled degrees of freedom are associated to the center-of-mass motion in the transverse space. The amount of supersymmetry provides for a 256-fold degeneracy in the continuum spectrum of the theory, which allows these states to be identified with the gravity multiplet. The SUSY might also allow for the existence of non-renormalization theorems that could lead to correct results for interactions.

It is crucial that each finite mass BPS object in M-Theory have an explicit description as some state in the matrix quantum mechanics. Furthermore, whenever BPS branes come together, or a 2-cycle which has a membrane wrapped around it shrinks to zero-size, an enhanced gauge symmetry must appear in the quantum dynamics.

The mechanism by which wrapped membranes and enhanced gauge symmetry appear in the model was introduced by Douglas [11] and elaborated upon in [6,14,8,15,16]. From the Kronheimer construction [23], the vertices of the extended Dynkin diagram are associated to the homology 2-cycles (which are $\mathbb{P}^{1} \mathrm{~s}$ ) of the ALE space. In [14], it was proposed that, in the blow-down, states which correspond to wrapped membranes exist on the Coulomb branch of the quiver gauge theory. In particular, a configuration describing a membrane wrapped on the $i^{\text {th }} \mathbb{P}^{1}$ would be described by a metastable state for which the hypermultiplets corresponding to that vertex vanished, $x_{i-1, i}=x_{i, i+1}=\cdots=0$. This picture was further discussed in [15], where a proposal was made for its extension to the blown-up ALE spaces.

A different approach was taken in [16], where it was proposed that the matrix description of membranes wrapped on these $\mathbb{P}^{1} \mathrm{~s}$ could be obtained by considering the ALE matrix models described above, but now relaxing the condition that $N_{i}=N k_{i}$. Instead, one should take

$$
N_{i}=N k_{i}+r_{i}
$$

where $N$ is chosen such that the set of $r_{i}>0$ are as small as possible. The resulting matrix model describes $N$ D0-branes propagating on a ALE space with $r_{i}$ membranes wrapped around the $i^{\text {th }} \mathbb{P}^{1}$. It is this latter approach which we will consider in the rest of the paper. 
If all of the $r_{i}=n k_{i}$ for some integer $n$ (we can include the possibility that $n=0$, in which case $N_{i}=N k_{i}$ ), then the corresponding homology 2-cycle is trivial, and the configuration of wrapped membranes can decay into a collection of D0-branes. A single wrapped membrane corresponds to a simple root of the spacetime gauge group, while the other roots have been conjectured to be given by bound states of these simple roots [11]. We will demonstrate this result explicitly in Section 3.

The objective of the following sections is to give a detailed account of several features of wrapped membranes in the ALE matrix models. We give a complete description of what BPS bound states of wrapped membranes form in the quantum mechanical system and then construct explicit bound state solutions for the $A_{n-1}$ series. We give a short discussion of the noncommutative geometry properties of the solutions and exhibit the spherical membrane properties for the explicit $A_{1}$ solution. For the $A_{1}$ ALE space, we examine the spectrum of excitations of the wrapped membrane, providing an explicit calculation of their energies. We find that the large $N$ behavior of the excitations matches supergravity expectations.

\section{Bound States of Wrapped Membranes}

We would like to determine the conditions under which a (non-threshold) BPS bound state of membranes can form. These are ground states of the interacting part of the theory that, classically, completely break the gauge symmetry group. Since the argument is classical, any solution of the F-terms for the hypermultiplets can be deformed to set the gauge fields equal to zero. In this way, the symmetry breaking pattern can be chosen to preserve the $S O(5)$ symmetry of the directions transverse to the ALE.

In order to determine the masses of the gauge fields, the argument can be extended to the six-dimensional quiver gauge theory. The Higgs mechanism requires that the extra degrees of freedom that give mass to a vector multiplet come from "eating" a hypermultiplet. The number of vector multiplets that can be Higgsed can be obtained by counting the total number of vector multiplet degrees of freedom $\left(4 \sum_{i} N_{i}^{2}\right)$ and subtracting the number of hypermultiplet $\left(4 \sum_{i, j \text { adj. }} N_{i} N_{j}\right)$ and decoupled $U(1)$ degrees of freedom (4). The resulting number must be smaller than zero or otherwise there remains a flat direction corresponding to a vector multiplet, in which case the membrane configuration can be separated classically into two separate bound states. We therefore need $N_{i}$ such that

$$
0 \geq 4\left(\sum_{i} N_{i}^{2}-\sum_{i, j \text { adj. }} N_{i} N_{j}-1\right)=2\left(\sum_{i, j} \hat{C}_{i j} N_{i} N_{j}-2\right)
$$


where $\hat{C}_{i j}$ is the extended Cartan matrix of the corresponding $A-D-E$ group. One immediately recognizes the quantity $\sum_{i, j} \hat{C}_{i j} N_{i} N_{j}$ as the squared norm of the vector $N_{i}$ in the lattice of the algebra. Since the inequality (3.1) is satisfied whenever $\sum_{i, j} \hat{C}_{i j} N_{i} N_{j} \leq 2$, we see that the state must either be neutral under the Cartan subalgebra (and is therefore a collection of D0-branes) or it is a root.

It is fairly straightforward, in general, to determine a bound on the energy of the state. Wrapped membrane bound states should form massive vector multiplets in seven spacetime dimensions. Since the fermionic zero-modes corresponding to the decoupled $U(1)$ center-ofmass motion already give rise to a 16-fold degeneracy, the ground state of the interacting part of the theory must be non-degenerate. This is difficult to prove in general, but we can still obtain a bound on the energy rather easily. We will then show that these states exist explicitly in the case of the $A_{n-1}$ ALE spaces.

Let us assume that the F-terms are zero, then, after computing the traces, the Hamiltonian for the D-terms we obtain from $(2.2)$ is

$$
H_{D}=\sum_{i}\left(\frac{N_{i} D_{i}^{2}}{2 g_{i}^{2}}-\frac{2 N_{i} d_{i} D_{i}}{g^{2}}\right) .
$$

Since the decoupled $U(1)$ is the sum of the $U(1)$ s at each vertex, the corresponding D-term, $D_{\text {dec. }}$, is given by the sum

$$
D_{\text {dec. }}=\frac{1}{\sum_{i} \frac{N_{i}}{g_{i}^{2}}} \sum_{i} \frac{N_{i} D_{i}}{g_{i}^{2}} .
$$

Therefore, each D-term has an expansion as

$$
D_{i}=D_{\text {dec. }}+D_{i, \text { int. }}
$$

where the $D_{i, \text { int. }}$ are orthogonal to each other and $D_{\text {dec. }}$ and satisfy

$$
\sum_{i} \frac{N_{i} D_{i, \text { int. }}}{g_{i}^{2}}=0
$$

Using the expansion (3.4) in (3.2), we find that the Hamiltonian for the decoupled $U(1)$ is

$$
H_{\text {dec. }}=D_{\text {dec. }} \sum_{i} N_{i}\left(\frac{D_{\text {dec. }}}{2 g_{i}^{2}}-\frac{2 d_{i}}{g^{2}}\right)
$$

This is minimized by

$$
D_{\text {dec. }}=-\frac{2 \sum_{i} N_{i} d_{i}}{g^{2} \sum_{i} \frac{N_{i}}{g_{i}^{2}}}
$$


which leads to a bound on the energy

$$
E \geq \frac{2\left(\sum_{i} N_{i} d_{i}\right)^{2}}{g^{4} \sum_{i} \frac{N_{i}}{g_{i}^{2}}}
$$

From this expression for the bound, we see that the D0-brane charge of the state is proportional to $\sum_{i} \frac{N_{i}}{g_{i}^{2}}$. If we take the $N_{i}$ as in (2.6) and apply (2.4), we find that

$$
E \geq \frac{2\left(\sum_{i} r_{i} d_{i}\right)^{2}}{g^{4} \sum_{i} \frac{N_{i}}{g_{i}^{2}}}
$$

A similar calculation works when the F-terms are non-zero. Defining $F_{\text {dec. }}$ in a manner analogous to (3.3), we find the solution

$$
F_{\text {dec. }}=-\frac{2 \sum_{i} N_{i} f_{i}}{g^{2} \sum_{i} \frac{N_{i}}{g_{i}^{2}}}
$$

Putting the results together, we find a bound

$$
E \geq \frac{2}{g^{4} \sum_{i} \frac{N_{i}}{g_{i}^{2}}}\left(\left(\sum_{i} r_{i} d_{i}\right)^{2}+\left|\sum_{i} r_{i} f_{i}\right|^{2}\right)=\frac{2\left|\sum_{i} r_{i} \vec{\zeta}_{i}\right|^{2}}{g^{4} \sum_{i} \frac{N_{i}}{g_{i}^{2}}}
$$

This is the light-cone energy of an object with mass

$$
m=\frac{2\left|\sum_{i} r_{i} \vec{\zeta}_{i}\right|}{g^{2}}
$$

and longitudinal momentum

$$
p^{+}=\sum_{i} \frac{N_{i}}{g_{i}^{2}}
$$

In particular, it is clear that by tuning the $g_{i}$, one assigns different amounts of longitudinal momentum to each simple root. In the DLCQ, the parameters responsible for this are the Wilson lines of the spacetime gauge group around the light-like circle.

In the large $N$ limit, we obtain from (3.11)

$$
E \geq \frac{2\left|\sum_{i} r_{i} \vec{\zeta}_{i}\right|^{2}}{N g^{2}}(1-O(1 / N))
$$

We will show explicitly below that there are solutions that satisfy the bound for any $N$. Moreover, from the DLCQ interpretation of the coupling constants, we know that at large $N$ the effects of the Wilson lines should disappear. In particular this implies that the ratios $g_{i} / g_{j}$ become unimportant in the large $N$ limit and therefore that some of these coupling 
constants decouple from the dynamics in the large $N$ limit. This can serve as a very useful calculational tool, and might serve to produce some non-renormalization theorems in the large $N$ limit.

As a check on our results, we can calculate the tension of the membrane from (3.12). For a single membrane state

$$
m=\frac{2|\vec{\zeta}|}{g^{2}}=T^{(2)} A,
$$

Since the area of the 2-sphere is $A=4 \pi|\vec{\zeta}|$, from (2.3) we recover (in string units) the well known result [25]

$$
T^{(2)}=\frac{T_{D 0}}{2 \pi}
$$

Now we will construct the bound states for all roots in the $A_{n-1}$ matrix models. First, as the $k_{i}=1$ for the $A_{n-1}$, the $r_{i}=0$ or 1 . For a state to be a root and non-trivial in homology, all of the $r_{i}=1$ must be adjacent and there must be at least one $r_{i}=0$.

We will begin with the simplifying assumption that the F-terms are set to zero. Later, we will describe how to obtain solutions when both $\mathrm{F}$ and D-terms are present. In order to solve the F-term equations of the hypermultiplets, we set all of the $a_{i}=0$ in the vector multiplets. To solve the F-term equations of the vector multiplets, we make the ansatz

$$
x_{i, i+1} y_{i+1, i}=y_{i+1, i} x_{i, i+1}=0 .
$$

Now we must minimize the D-terms. The states we are interested in should be excited states under the decoupled $U(1)$, but should form supersymmetric bound states of the internal part of the theory. Therefore, we will set $D_{i, \text { int. }}=0$ and seek a solution to the equations

$$
\bar{x}_{i-1, i} x_{i-1, i}-y_{i, i-1} \bar{y}_{i, i-1}-x_{i, i+1} \bar{x}_{i, i+1}+\bar{y}_{i+1, i} y_{i+1, i}=\left(2 d_{i}+\frac{g^{2}}{g_{i}^{2}} D_{\text {dec. }}\right) \mathbb{1}_{N_{i}} .
$$

With our ansatz, the operators $\bar{x}_{i-1, i} x_{i-1, i}$ and $y_{i, i-1} \bar{y}_{i, i-1}$ commute. As they are selfadjoint, they can be simultaneously diagonalized, and they moreover multiply to zero. Therefore they are fully determined as the projections to the positive and negative spectra of the operator

$$
O_{i}=\bar{x}_{i-1, i} x_{i-1, i}-y_{i, i-1} \bar{y}_{i, i-1}
$$

Now, $O_{i}$ and the operator

$$
O_{i}^{\prime}=x_{i-1, i} \bar{x}_{i-1, i}-\bar{y}_{i, i-1} y_{i, i-1}
$$


which appears in the D-term at the $(i-1)^{t h}$ vertex, are isospectral, except for an extra zero eigenvalue on one of them whenever $r_{i}=r_{i-1} \pm 1$, so that $N_{i}=N_{i-1} \pm 1$. Imposing an ordering on the eigenvalues, $O_{i}=\operatorname{diag}\left(O_{i}^{1}, \ldots, O_{i}^{N_{i}}\right)$, with $O_{i}^{1} \geq \cdots \geq O_{i}^{N_{i}}$, the D-term equations (3.18) become

$$
O_{i}^{\ell}-O_{i+1}^{\prime \ell}=\left(2 d_{i}+\frac{g^{2}}{g_{i}^{2}} D_{\text {dec. }}\right)
$$

Now, if the state is nontrivial, then, without loss of generality, we can label the first vertex where there is a membrane wrapped as the $0^{\text {th }}$, so that on the quiver diagram we will have the sequence $\ldots, r_{n-1}=0, r_{0}=1, r_{1}=1, \ldots, r_{\iota}=1, r_{\iota+1}=0, \ldots$ (i.e., there are $\iota 1 \mathrm{~s}$ and $n-\iota 0 \mathrm{~s}$ on the diagram). Then $O_{0}^{N+1}=0$, so that (3.21) can be solved to find $O_{1}^{\prime N+1}=-\left(2 d_{0}+\frac{g^{2}}{g_{0}^{2}} D_{\text {dec. }}\right)$. But then $O_{1}^{N+1}=-\left(2 d_{0}+\frac{g^{2}}{g_{0}^{2}} D_{\text {dec. }}\right)$ as well. By repeated use of (3.21), we can follow these eigenvalues until we get to the transition $r_{\iota}=1 \rightarrow r_{\iota+1}=0$, where we jump to the next non-zero eigenvalue, so that

$$
O_{\iota+1}^{N}=O_{\iota+1}^{\prime N+1}=-\sum_{j=1}^{\iota+1}\left(2 d_{j-1}+\frac{g^{2}}{g_{j-1}^{2}} D_{\text {dec. }}\right) .
$$

By iterating this process around to the $0^{\text {th }}$ vertex, we find

$$
\begin{aligned}
& O_{0}^{N}=-\sum_{j=1}^{n}\left(2 d_{j-1}+\frac{g^{2}}{g_{j-1}^{2}} D_{\text {dec. }}\right)=-D_{\text {dec }} \\
& O_{1}^{N}=-2 d_{0}-\left(1+\frac{g^{2}}{g_{0}^{2}}\right) D_{\text {dec. }} .
\end{aligned}
$$

By continuing this process of circuiting the diagram, we determine all of the eigenvalues. We find the general expression

$$
O_{k}^{\ell}=-\sum_{j=1}^{k}\left(2 d_{j-1}+\frac{g^{2}}{g_{j-1}^{2}} D_{\text {dec. }}\right)- \begin{cases}(N+1-\ell) D_{\text {dec. }} & \text { for } 0 \leq k \leq \iota \\ (N-\ell) D_{\text {dec. }} & \text { for } \iota<k<n\end{cases}
$$

The explicit form for $D_{\text {dec. }}$ is given by the solution (3.7). For $n=1$, this formula is in agreement with the $A_{1}$ solution presented in [16].

Now let us consider the situation when both $\mathrm{F}$ and D-terms are present. Here, in addition to solving the D-terms (3.18), we must also solve the F-term equations, which are of the form

$$
y_{i, i-1} x_{i-1, i}-x_{i, i+1} y_{i+1, i}=\left(2 f_{i}+\frac{g^{2}}{g_{i}^{2}} F_{\text {dec. }}\right) \mathbb{1}_{N_{i}} .
$$

The simplest ansatz for a solution would clearly be one for which the above method of tracing eigenvalues around the quiver diagram would work. This will require that the individual 
terms in (3.18) and (3.25) commute amongst themselves, so that they are simultaneously diagonalizable. We must therefore demand that

$$
\begin{aligned}
& \bar{y}_{i, i-1} y_{i, i-1} x_{i-1, i}=x_{i-1, i} y_{i, i-1} \bar{y}_{i, i-1} \\
& y_{i, i-1} x_{i-1, i} \bar{x}_{i-1, i}=\bar{x}_{i-1, i} x_{i-1, i} y_{i, i-1} .
\end{aligned}
$$

If we then define

$$
\begin{aligned}
& P_{i}=y_{i, i-1} x_{i-1, i} \\
& P_{i}^{\prime}=x_{i-1, i} y_{i, i-1},
\end{aligned}
$$

then it is a straightforward exercise to show that

$$
\left[O_{i}, P_{i}\right]=\left[P_{i}, \bar{P}_{i}\right]=0
$$

From (3.26), we see that $x_{i-1, i}$ generates a sort of "supersymmetry" for the matrices $\bar{y}_{i, i-1} y_{i, i-1}$ and $y_{i, i-1} \bar{y}_{i, i-1}$. That is, it acts as an intertwiner between their eigenspaces, so that given an eigenstate, $|\alpha\rangle$, of $y_{i, i-1} \bar{y}_{i, i-1}$, the state $x_{i}|\alpha\rangle$ is an eigenstate of $\bar{y}_{i, i-1} y_{i, i-1}$ with the same eigenvalue. Similarly, $y_{i, i-1}$ is a supersymmetry for $x_{i-1, i} \bar{x}_{i-1, i}$ and $\bar{x}_{i-1, i} x_{i-1, i}$, while both $x_{i-1, i}$ and $y_{i, i-1}$ are supersymmetries for $P_{i}$ and $P_{i}^{\prime}$. We can therefore conclude that, as before, $O_{i}$ and $O_{i}^{\prime}$ have the same spectrum, except, perhaps, for a zero-mode. Similarly, the spectra of $P_{i}$ and $P_{i}^{\prime}$ can differ only by a zero-mode.

Solutions may be explicitly obtained by solving the eigenvalue equations (3.21) and

$$
P_{i}^{\ell}-P_{i+1}^{\prime \ell}=\left(2 f_{i}+\frac{g^{2}}{g_{i}^{2}} F_{\text {dec. }}\right)
$$

via following the eigenvalues around the quiver diagram, as outlined above. Then we find that the $O_{i}$ are given by (3.24), as before, while the

$$
P_{k}^{\ell}=-\sum_{j=1}^{k}\left(2 f_{j-1}+\frac{g^{2}}{g_{j-1}^{2}} F_{\text {dec. }}\right)- \begin{cases}(N+1-\ell) F_{\text {dec. }} & \text { for } 0 \leq k \leq \iota \\ (N-\ell) F_{\text {dec. }} & \text { for } \iota<k<n,\end{cases}
$$

where $F_{\text {dec. }}$ is given by $(3.10)$. We note that if the F-terms are in fact absent, we recover our old ansatz (3.17).

Within the ansatz (3.26) the solutions obtained from (3.24) and (3.30) are unique. Explicit expressions for the $x_{i, i+1}$ and $y_{i+1, i}$ can be obtained by choosing, say, the $x_{i, i+1}$ to have positive real entries. This can always be achieved by a gauge transformation. The solution obtained in this manner is a classical solution to the equations for a supersymmetric vacuum (of the internal theory), and therefore there is a corresponding quantum mechanical state whose wavefunction is localized near the classical solution. It is clear that all of the hypermultiplets have a mass gap and it is reasonable to believe that the solution breaks the gauge group completely. 


\section{Noncommutative Geometry and Spherical Membranes}

It is worthwhile to illustrate the geometric nature of the membrane solutions we have constructed. We will show in this section that the natural set of gauge-invariant coordinates derived from the quiver theory are elevated by these solutions to noncommuting coordinates $^{1}$. These noncommuting coordinates will satisfy the constraints imposed by the ALE space geometry to leading order in the $1 / N$ expansion.

In the case of the $A_{n-1}$, we know that $\mathbb{Z}_{n}$-invariant coordinates are given by invariant products of the coordinates of the $\mathbb{C}^{n}$ being quotiented. In terms of the quiver theory describing a single membrane wrapped on the $0^{\text {th }} \mathbb{P}^{1}$, we can consider the $N_{0} \times N_{0}$ matrices

$$
\begin{aligned}
U & =x_{01} \cdots x_{n-1,0} \\
V & =y_{0, n-1} \cdots y_{10} \\
W & =x_{01} y_{10} .
\end{aligned}
$$

Coordinates $(u, v, w)$ on an ALE space of the same shape will satisfy

$$
u v=P(w)
$$

where $P(w)$ is an $n^{\text {th }}$-order polynomial whose coefficients are determined by the $f_{i}$.

Now, when the $f_{i}=0$ (e.g. on the blow-down), $P(w)=w^{n}$. Also, from (3.30) we see that $W=0$. Since (3.24) tells us that $O_{0}$ is positive-definite (for $\sum_{i} r_{i} d_{i}>0, D_{\text {dec. }}$ is negative-definite), we know that $\bar{y}_{01} y_{01}=0$, whence $y_{01}=V=0$. On the other hand, $O_{1}$ is negative-definite, so that $x_{01} \bar{x}_{01}=0$ and $x_{01}=U=0$ as well. Therefore the membrane is indeed localized at the singularity, as $u=v=w=0$ is the singular locus of (4.2).

By means of the F-term equations (3.25), one can show that for large $N$

$$
U V=P(W)+O(1 / N)
$$

Moreover, one can also show that

$$
\begin{aligned}
& {[W, U]=F_{\text {dec. }} U=-\frac{2 \sum_{i} d_{i} f_{i}}{N} U+O\left(1 / N^{2}\right)} \\
& {[W, V]=-F_{\text {dec. }} V=\frac{2 \sum_{i} d_{i} f_{i}}{N} V+O\left(1 / N^{2}\right) .}
\end{aligned}
$$

This is reminiscent of the angular momentum commutation relations, and shows that the intrinsic geometry of the wrapped membranes that we have constructed is noncommutative. The membranes are spherical, but if we were to probe the membrane locally, where it approaches the flat membrane solution, we would find an effective "Planck constant" of order

\footnotetext{
${ }^{1}$ For a recent discussion of noncommutative geometry and matrix theory, see [26] and references therein.
} 
$1 / N$ times the area of the wrapped membrane. This comes as no surprise when we consider the way membranes were first constructed in [17,1], as well as the discussion of spherical membranes in matrix theory by Kabat and Taylor [18].

As an illustration of the noncommutative properties of the solutions, it is illuminating to check that single membranes have properties which are analogous to those discussed in [18]. In the $A_{1}$ case, we can set the F-terms to zero and use (3.24) to solve for the hypermultiplets. Then $X_{01}=Y_{01}=0$, while the other hypermultiplet components are off-diagonal (up to a $U(N) \times U(N+1)$ gauge transformation)

$$
\begin{gathered}
X_{10}=\sqrt{\frac{4 d}{2 N+1}}\left(\begin{array}{ccccc}
\sqrt{N} & 0 & \cdots & \cdots & 0 \\
0 & \sqrt{N-1} & 0 & \cdots & \vdots \\
\vdots & 0 & \ddots & 0 & \vdots \\
0 & \cdots & 0 & 1 & 0
\end{array}\right) \\
Y_{10}=\sqrt{\frac{4 d}{2 N+1}}\left(\begin{array}{ccccc}
0 & 1 & 0 & \cdots & 0 \\
\vdots & 0 & \ddots & 0 & \vdots \\
\vdots & \cdots & 0 & \sqrt{N-1} & 0 \\
0 & \cdots & \cdots & 0 & \sqrt{N}
\end{array}\right)
\end{gathered}
$$

where we have set $g_{0}=g_{1}$ for convenience. As coordinates on the ALE space are gaugeinvariant combinations of the quiver theory hypermultiplet components, we may consider the quadratic combinations

$$
\begin{aligned}
& J_{0}=\frac{1}{8 \sqrt{d}}\left(X_{10} \bar{X}_{10}-Y_{10} \bar{Y}_{10}\right) \\
& =\frac{\sqrt{d}}{2 N+1}\left(\begin{array}{ccccc}
N-1 & 0 & \cdots & \cdots & 0 \\
0 & N-3 & 0 & \cdots & \vdots \\
\vdots & 0 & \ddots & 0 & \vdots \\
\vdots & \cdots & 0 & -(N-3) & 0 \\
0 & \cdots & \cdots & 0 & -(N-1)
\end{array}\right) \\
& J_{+}=\frac{1}{4 \sqrt{d}} Y_{10} \bar{X}_{10}=\frac{2 \sqrt{d}}{2 N+1}\left(\begin{array}{ccccc}
0 & \sqrt{N-1} & 0 & \cdots & 0 \\
\vdots & 0 & \sqrt{2(N-2)} & 0 & \vdots \\
\vdots & \cdots & 0 & \ddots & 0 \\
\vdots & \cdots & \ldots & 0 & \sqrt{N-1} \\
0 & \cdots & \cdots & \cdots & 0
\end{array}\right)
\end{aligned}
$$




$$
J_{-}=\frac{1}{4 \sqrt{d}} X_{10} \bar{Y}_{10}=\frac{2 \sqrt{d}}{2 N+1}\left(\begin{array}{ccccc}
0 & \cdots & \cdots & \cdots & 0 \\
\sqrt{N-1} & 0 & \cdots & \cdots & \vdots \\
0 & \sqrt{2(N-2)} & 0 & \cdots & \vdots \\
\vdots & 0 & \ddots & 0 & \vdots \\
0 & \cdots & 0 & \sqrt{N-1} & 0
\end{array}\right) .
$$

These matrices are proportional to the generators of the $\mathbf{N}$ of $S U(2)$, so they commute according to the $S U(2)$ algebra

$$
\begin{aligned}
& {\left[J_{+}, J_{-}\right]=2\left(\frac{2 \sqrt{d}}{2 N+1}\right) J_{0}} \\
& {\left[J_{ \pm}, J_{0}\right]=\mp\left(\frac{2 \sqrt{d}}{2 N+1}\right) J_{ \pm}}
\end{aligned}
$$

and the sum over their squares is

$$
\sum_{i} J_{i}^{2}=J_{0}^{2}+\frac{1}{2}\left\{J_{+}, J_{-}\right\}=\frac{4\left(N^{2}-1\right) d}{(2 N+1)^{2}} \mathbb{1}_{N}=r^{2} \mathbb{1}_{N} .
$$

Evidently, (4.6)-(4.8) are the redundant set of coordinates which parameterize the surface of the membrane. We note that the set of operators $\tilde{J}_{+}=\bar{X}_{10} Y_{10} / 4 \sqrt{d}, \ldots$ also form a set of membrane coordinates, this time in the $\mathbf{N}+\mathbf{1}$ representation. That there is a pair of good membrane coordinates can be useful in calculations.

As in [18], we can ask if there is a regime in which these membranes approach the flat membrane solution. At large $N$,

$$
\left[J_{+}, J_{-}\right]=\frac{d}{N}\left(\begin{array}{cc}
\mathbb{1} & 0 \\
0 & -\mathbb{1}
\end{array}\right)+O\left(1 / N^{2}\right),
$$

which, when restricted to the upper-left quadrant, resembles the commutation relations of the coordinates of the flat membrane. In the semi-classical correspondence between Poisson brackets and commutators of $[17,1]$, we find the "Planck constant"

$$
\hbar=\frac{d}{N},
$$

which, as promised above, is proportional to the area of the membrane.

In the large $N$ limit, from (4.10), we also find that $r$ is the radius of the membrane,

$$
r=\sqrt{d}\left(1-\frac{1}{2 N}\right) .
$$


Our choice of normalization in (4.6)-(4.8) was made specifically to obtain this leading-order behavior. These results agree nicely with those of [18], once the different $N$ dependence of the membrane longitudinal momentum here is taken into account. We note that the corrections to the radius (4.13) are stronger than those found for the membranes in [18], being of $O(1 / N)$ as compared to $O\left(1 / N^{2}\right)$.

\section{The Spectrum of Excitations of the Wrapped Membrane}

We will now concentrate our efforts on finding the spectrum of excitations around the membrane solution for the simplest possible case, namely the $A_{1}$ singularity. We will first examine a toy model, in order to establish a framework for understanding the problem. Then we utilize the rotational invariance of the $A_{1}$ solution to determine the allowed representations for perturbations around the membrane. We then calculate the mass spectrum for the P-wave excitations and, finally, for all modes.

We note that the perturbative approach we take to the calculation of the spectrum of fluctuations is well-suited only for membranes which are large as compared to the 11dimensional Planck length. It is only in this limit that we may reliably expect that the fluctuations have energies small compared to the mass of the membrane and are thereby long-lived.

We want to describe the linearized spectrum of excitations of a large membrane wrapped around a sphere. Consider a toy model consisting of a massless free scalar field on the space $S^{2} \times \mathbb{R}$, where $\mathbb{R}$ is time and the sphere is of radius $r$. It is a straightforward exercise to show that the normal modes are the spherical harmonics, with masses given by

$$
M_{\ell}=\frac{\sqrt{\ell(\ell+1)}}{r}
$$

for $\ell=0,1$, etc. This toy model reproduces the linearized degrees of freedom for a BPS membrane wrapped on a 2 -sphere, if we give the action maximal supersymmetry. Instead of a single scalar, we can consider $U(1)$ SYM with maximal supersymmetry as our starting point. This is the natural effective worldvolume theory of a D2-brane (see, e.g., [27,28]) and, in particular, is valid for the large radius limit we are considering.

As we are considering a free $U(1)$ theory, there is no need to quantize the magnetic flux through the sphere. This quantum number is associated to the compactness of the 11th dimension. In order to get an explicitly $O(6)$-invariant Lagrangian, as one would expect from M-Theory, one can dualize the vector into a scalar.

Since half of the supersymmetries are broken in the ALE space, we must also explicitly break half of the supersymmetry of our toy model. This can be achieved by twisting one 
complex scalar. In particular, we identify one of the scalars as a section of the normal bundle of the 2-sphere sitting in the ALE space. The resulting theory is supersymmetric if the fermions are sections of the bundle $\mathcal{O}(-1) \oplus \mathcal{O}(1)$. There are six bosonic zero modes, which correspond to the motion of the membrane in the transverse $\mathbb{R}^{6}$. The corresponding eight fermionic zero modes generate the required 16-fold degeneracy of the ground state. For the massive modes, there are eight bosonic and eight fermionic modes for each spherical harmonic. This gives us the information we require in order to interpret the calculation we will now make in the matrix model.

For the $A_{1}$ singularity there is an $S U(2)$ rotational invariance which aids in the solution. Namely, since there is a pair of hypermultiplets connecting the same two vertices, the action (and so too the D and F-terms) is invariant under the $S U(2)_{r}$ transformation

$$
\left(\begin{array}{l}
x_{01} \\
y_{01}
\end{array}\right) \rightarrow\left(\begin{array}{l}
x_{01}^{\prime} \\
y_{01}^{\prime}
\end{array}\right)=R\left(\begin{array}{l}
x_{01} \\
y_{01}
\end{array}\right), \quad\left(\begin{array}{l}
x_{10} \\
y_{10}
\end{array}\right) \rightarrow\left(\begin{array}{l}
x_{10}^{\prime} \\
y_{10}^{\prime}
\end{array}\right)=R\left(\begin{array}{l}
x_{10} \\
y_{10}
\end{array}\right)
$$

for $R \in S U(2)_{r}$, which mixes the hypermultiplets. Clearly there is a $U(N) \times U(N+1)$ gauge transformation,

$$
\left(\begin{array}{cc}
U & 0 \\
0 & U
\end{array}\right) R\left(\begin{array}{c}
X_{10} \\
Y_{10}
\end{array}\right) V=\left(\begin{array}{c}
X_{10} \\
Y_{10}
\end{array}\right)
$$

where $U \in U(N)$ and $V \in U(N+1)$, that undoes the action of (5.2) on the membrane ground state (4.5). In other words, the solution (4.5) is invariant under the product $U \otimes R \otimes V$, which is a consequence of the fact that the ground state is spherically symmetric. Obviously $U$ and $V$ must themselves correspond to (inverse) elements of $S U(2)_{r}$.

Now the action of $U \otimes R \otimes V$ on any perturbations around (4.5) should be faithful. Since $R$ acts as the $\mathbf{2}$ of $S U(2)_{r}$ on $\left(\begin{array}{l}\delta x_{10} \\ \delta y_{10}\end{array}\right)$ and $U$ and $V$ act as the $\mathbf{N}$ and $\mathbf{N}+\mathbf{1}$, respectively, $U \otimes R \otimes V$ must act as the representation

$$
\mathbf{2} \otimes \mathbf{N} \otimes(\mathbf{N}+\mathbf{1})=\mathbf{1} \oplus \mathbf{3} \oplus \mathbf{3} \oplus \mathbf{5} \oplus \mathbf{5} \oplus \cdots \oplus \mathbf{2} \mathbf{N}-\mathbf{1} \oplus \mathbf{2 N}-\mathbf{1} \oplus \mathbf{2} \mathbf{N}+\mathbf{1}
$$

On the other hand, under this gauge transformation, the vector multiplets transform in the

$$
\begin{aligned}
\mathbf{N} \otimes \mathbf{N} & =\mathbf{1} \oplus \mathbf{3} \oplus \cdots \oplus \mathbf{2} \mathbf{N}-\mathbf{1} \\
(\mathbf{N}+\mathbf{1}) \otimes(\mathbf{N}+\mathbf{1}) & =\mathbf{1} \oplus \mathbf{3} \oplus \cdots \oplus \mathbf{2} \mathbf{N}+\mathbf{1}
\end{aligned}
$$

representations.

One immediately notices that the dimension of the representation of the vectors (5.5) is greater by a singlet than that of the hypermultiplets in (5.4). This singlet is simply the 
decoupled $U(1)$. The absence of a singlet for the decoupled $U(1)$ in the hypermultiplet representation protects against the possibility of Higgsing away this element of the gauge symmetry. Another important observation about (5.4) and (5.5) is that only even spherical harmonics will appear in the multipole expansion of the perturbations. This is a considerable simplification, and we will be able to compute the full spectrum of excitations of the wrapped membrane.

As a start, let us now calculate the mass spectrum for the P-wave excitations, i.e., those in the 3 of $S U(2)_{r}$. The calculation will be illustrative of the techniques we will use to compute the spectrum for generic $j$.

Since the masses are generated via the Higgs mechanism, it will be sufficient to calculate the masses of the vector multiplets, since these are related to those of the hypers in an obvious way. Furthermore, we can exploit the $S O(5)$ invariance of the scalars in the vector multiplet, so that we have $a_{0 i}=a_{0}^{\alpha} \lambda_{\alpha}^{(N+1)}, a_{1 i}=a_{1}^{\alpha} \lambda_{\alpha}^{(N)}$, for each $i$, where the $\lambda_{\alpha}^{(N)}$ generate the $\mathbf{N}$ of $S U(2)_{r}$. Finally, we note from the $S U(2)_{r}$ invariance that we can take $a_{0}^{\alpha}=a_{0}$, $a_{1}^{\alpha}=a_{1}$, for each $\alpha$. We then can compute the mass of $a_{0}$ and $a_{1}$ for the simplest case, namely the Cartan generator, $\lambda_{0}$.

The quadratic part of the Lagrangian for $a_{0}$ and $a_{1}$ is

$$
\begin{aligned}
\mathcal{L}= & \frac{1}{2 g_{0}^{2}} \operatorname{Tr}\left(\lambda_{0}^{(N+1)}\right)^{2} \dot{a}_{0}^{2}+\frac{1}{2 g_{1}^{2}} \operatorname{Tr}\left(\lambda_{0}^{(N)}\right)^{2} \dot{a}_{1}^{2} \\
& -\frac{1}{g^{2}}\left(\operatorname{Tr}\left[\left(\lambda_{0}^{(N+1)}\right)^{2}\left(\bar{X}_{10} X_{10}+\bar{Y}_{10} Y_{10}\right)\right] a_{0}^{2}+\operatorname{Tr}\left[\left(\lambda_{0}^{(N)}\right)^{2}\left(X_{10} \bar{X}_{10}+Y_{10} \bar{Y}_{10}\right)\right] a_{1}^{2}\right. \\
& \left.+2 \operatorname{Tr}\left[\lambda_{0}^{(N)} X_{10} \lambda_{0}^{(N+1)} \bar{X}_{10}+\lambda_{0}^{(N)} Y_{10} \lambda_{0}^{(N+1)} \bar{Y}_{10}\right] a_{0} a_{1}\right) .
\end{aligned}
$$

From (4.5), if we generalize to arbitrary coupling constants at each vertex, we have

$$
\begin{aligned}
& X_{10} \bar{X}_{10}+Y_{10} \bar{Y}_{10}=(N+1)\left|D_{\text {dec. }}\right| \mathbb{1}_{N} \\
& \bar{X}_{10} X_{10}+\bar{Y}_{10} Y_{10}=N\left|D_{\text {dec. }}\right| \mathbb{1}_{N+1} \\
& X_{10} \bar{X}_{10}-Y_{10} \bar{Y}_{10}=\left|D_{\text {dec. }}\right| \lambda_{0}^{(N)} \\
& \bar{X}_{10} X_{10}-\bar{Y}_{10} Y_{10}=\left|D_{\text {dec. }}\right| \lambda_{0}^{(N+1)} .
\end{aligned}
$$

To simplify the cross-terms in (5.6), we use the "commutation" relations

$$
\begin{aligned}
\lambda_{0}^{(N)} X_{10}-X_{10} \lambda_{0}^{(N+1)}+X_{10} & =0 \\
\lambda_{0}^{(N)} Y_{10}-Y_{10} \lambda_{0}^{(N+1)}-Y_{10} & =0 .
\end{aligned}
$$

These relations encode, in part, the invariance of the membrane solution (4.5) under the rotation group of the sphere (5.2). One may also readily calculate

$$
\operatorname{Tr}\left(\lambda_{0}^{(N)}\right)^{2}=\frac{(N-1) N(N+1)}{3} .
$$


Using these relations in (5.6) yields

$$
\mathcal{L}=\frac{1}{2}\left(\dot{\tilde{a}}_{0}{ }^{2}+\dot{\tilde{a}}_{1}{ }^{2}\right)-\frac{\left|D_{\text {dec. }}\right|}{g^{2}}\left[N g_{0}^{2} \tilde{a}_{0}^{2}+2 \sqrt{(N-1)(N+2)} g_{0} g_{1} \tilde{a}_{0} \tilde{a}_{1}+(N+1) g_{1}^{2} \tilde{a}_{1}^{2}\right]
$$

where we have defined the normalized variables

$$
\begin{aligned}
& \tilde{a}_{0}=\sqrt{\operatorname{Tr}\left(\lambda_{0}^{(N+1)}\right)^{2}} \frac{a_{0}}{g_{0}} \\
& \tilde{a}_{1}=\sqrt{\operatorname{Tr}\left(\lambda_{0}^{(N)}\right)^{2}} \frac{a_{1}}{g_{1}} .
\end{aligned}
$$

The eigenvalues of the mass matrix are the energies for the oscillator modes

$$
\begin{aligned}
\omega_{ \pm}^{2} & =\left[\left(N g_{0}^{2}+(N+1) g_{1}^{2}\right) \pm \sqrt{\left(N g_{0}^{2}+(N+1) g_{1}^{2}\right)^{2}-8 g_{0}^{2} g_{1}^{2}}\right] \frac{\left|D_{\text {dec. }}\right|}{g^{2}} \\
& \sim \frac{2 g_{0}^{2} g_{1}^{2} d}{g^{4}}\left[1 \pm\left(1-\frac{4 g^{4}}{g_{0}^{2} g_{1}^{2}} \frac{1}{N^{2}}+\cdots\right)\right] .
\end{aligned}
$$

Therefore, in the large $N$ limit, we find two modes, one whose energy is independent of $N$ at leading order (and therefore decouples) and another whose energy is

$$
\omega_{-}=\frac{2 \sqrt{2 d}}{N}
$$

and survives as the membrane excitation as $N \rightarrow \infty$. Notice that the energy of this mode only depends on $d$, providing some evidence for the hypothesis made in Section 3 that the ratio $g_{0} / g_{1}$ decouples in the large $N$ limit . The mass of this mode, $\delta m$, can be calculated from

$$
\omega_{-}=\frac{(m+\delta m)^{2}-m^{2}}{2 p^{+}} \sim \frac{m \delta m}{p^{+}}
$$

where $m$ and $p^{+}$are given by (3.12) and (3.13), respectively. In this way, we find

$$
\delta m=\sqrt{\frac{2}{d}}=\frac{\sqrt{2}}{r}
$$

where $r$ is the radius of the membrane. This result agrees precisely with our toy model result (5.1) for the $\mathrm{P}$-wave, $\ell=1$.

Now, for generic excitations appearing in the $\mathbf{2} \mathbf{j}+\mathbf{1}$ representation under the decomposition (5.4), we may again exploit symmetry arguments. In general, these reduce the problem to computing the masses of the scalars in the vector multiplets, which may be taken to be of the form

$$
\begin{aligned}
& a_{0 i}=a_{0}\left(\lambda_{+}^{(N+1)}\right)^{j}+\bar{a}_{0}\left(\lambda_{-}^{(N+1)}\right)^{j} \\
& a_{1 i}=a_{1}\left(\lambda_{+}^{(N)}\right)^{j}+\bar{a}_{1}\left(\lambda_{-}^{(N)}\right)^{j},
\end{aligned}
$$


for each $i=1, \ldots, 5$. As a motivation for why we have chosen this particular form, we note that these are the highest weight states for their respective $S U(2)_{r}$ representations. This stems from the fact that, as the representations are irreducible, all of the operators acting in a given representation are constructed from polynomials in the generators.

After dropping the terms which vanish after taking traces, the quadratic Lagrangian for these modes is

$$
\begin{aligned}
\mathcal{L}= & 2 \operatorname{Tr}\left[\left(\lambda_{+}^{(N+1)}\right)^{j}\left(\lambda_{-}^{(N+1)}\right)^{j}\right]\left(\frac{1}{g_{0}^{2}}\left|\dot{a}_{0}\right|^{2}-\frac{N\left|D_{\text {dec. }}\right|}{g^{2}}\left|a_{0}\right|^{2}\right) \\
& +2 \operatorname{Tr}\left[\left(\lambda_{+}^{(N)}\right)^{j}\left(\lambda_{-}^{(N)}\right)^{j}\right]\left(\frac{1}{g_{1}^{2}}\left|\dot{a}_{1}\right|^{2}-\frac{(N+1)\left|D_{\text {dec. }}\right|}{g^{2}}\left|a_{1}\right|^{2}\right) \\
& -\frac{2}{g^{2}} a_{0} \bar{a}_{1}\left[\left(\lambda_{+}^{(N)}\right)^{j} X_{10}\left(\lambda_{-}^{(N+1)}\right)^{j} \bar{X}_{10}+\left(\lambda_{+}^{(N)}\right)^{j} Y_{10}\left(\lambda_{-}^{(N+1)}\right)^{j} \bar{Y}_{10}\right] \\
& -\frac{2}{g^{2}} \bar{a}_{0} a_{1}\left[\left(\lambda_{-}^{(N)}\right)^{j} X_{10}\left(\lambda_{+}^{(N+1)}\right)^{j} \bar{X}_{10}+\left(\lambda_{-}^{(N)}\right)^{j} Y_{10}\left(\lambda_{+}^{(N+1)}\right)^{j} \bar{Y}_{10}\right] .
\end{aligned}
$$

The cross-terms here can be simplified through the use of the "commutation" relations

$$
\begin{array}{r}
X_{10} \lambda_{+}^{(N+1)}-\lambda_{+}^{(N)} X_{10}-Y_{10}=0 \\
X_{10} \lambda_{-}^{(N+1)}-\lambda_{-}^{(N)} X_{10}=0 \\
Y_{10} \lambda_{+}^{(N+1)}-\lambda_{+}^{(N)} Y_{10}=0 \\
Y_{10} \lambda_{-}^{(N+1)}-\lambda_{-}^{(N)} Y_{10}-X_{10}=0
\end{array}
$$

As in the case of the relations (5.8), these encode the rotational invariance of the membrane solution. By repetitive application of (5.18), one may establish the useful relationship

$$
(N-j) \operatorname{Tr}\left[\left(\lambda_{+}^{(N+1)}\right)^{j}\left(\lambda_{-}^{(N+1)}\right)^{j}\right]=(N+j+1) \operatorname{Tr}\left[\left(\lambda_{+}^{(N)}\right)^{j}\left(\lambda_{-}^{(N)}\right)^{j}\right] .
$$

In terms of the normalized variables

$$
\begin{aligned}
& \tilde{a}_{0}=\sqrt{2 \operatorname{Tr}\left[\left(\lambda_{+}^{(N+1)}\right)^{j}\left(\lambda_{-}^{(N+1)}\right)^{j}\right]} \frac{a_{0}}{g_{0}} \\
& \tilde{a}_{1}=\sqrt{2 \operatorname{Tr}\left[\left(\lambda_{+}^{(N)}\right)^{j}\left(\lambda_{-}^{(N)}\right)^{j}\right]} \frac{a_{1}}{g_{1}},
\end{aligned}
$$

the Lagrangian (5.17) becomes

$$
\begin{aligned}
\mathcal{L}=\left|\dot{\tilde{a}}_{0}\right|^{2}+\left|\tilde{\tilde{a}}_{1}\right|^{2}-\frac{\left|D_{\text {dec. }}\right|}{g^{2}}\left(N g_{0}^{2}\left|\tilde{a}_{0}\right|^{2}+(N+1) g_{1}^{2}\left|\tilde{a}_{1}\right|^{2}\right) \\
-\frac{\left|D_{\text {dec. }}\right|}{g^{2}} \sqrt{(N-j)(N+j+1)} g_{0} g_{1}\left(\tilde{a}_{0} \overline{\tilde{a}}_{1}+\overline{\tilde{a}}_{0} \tilde{a}_{1}\right),
\end{aligned}
$$


From this, we find the energies of the modes as the eigenvalues of the mass matrix

$$
\begin{aligned}
\omega_{ \pm}^{2} & =\left[\left(N g_{0}^{2}+(N+1) g_{1}^{2}\right) \pm \sqrt{\left(N g_{0}^{2}+(N+1) g_{1}^{2}\right)^{2}-4 j(j+1) g_{0}^{2} g_{1}^{2}}\right] \frac{\left|D_{\text {dec. }}\right|}{g^{2}} \\
& \sim \frac{2 g_{0}^{2} g_{1}^{2} d}{g^{4}}\left[1 \pm\left(1-\frac{2 j(j+1) g^{4}}{g_{0}^{2} g_{1}^{2}} \frac{1}{N^{2}}+\cdots\right)\right] .
\end{aligned}
$$

We note that, in the case $j=1$, this reduces properly to (5.12).

As in the case of the $\mathrm{P}$-waves above, we can consider the behavior at large $N$. There are again two modes, one which decouples and another with energy

$$
\omega_{-}=\frac{\sqrt{2 j(j+1) d}}{N}
$$

and mass

$$
\delta m=\sqrt{\frac{j(j+1)}{d}}=\frac{\sqrt{j(j+1)}}{r},
$$

which agrees exactly with the toy model result (5.1). In the limit of large radius, the effective field theory on the membrane and the perturbation expansion in the matrix model agree.

\section{Conclusions}

We have given quite a bit of evidence that the matrix model proposed in $[19,16]$ captures many of the essential features of M-Theory membranes which are wrapped around the homology 2-cycles of an ALE space. In [16], we gave an explicit derivation of the solution describing the wrapped membrane in the $A_{1}$ case. We found that its energy, as well as the leading order membrane-antimembrane potential, had the properties necessary for its interpretation as a wrapped membrane.

In Section 3, we gave a counting argument that set the correspondence between BPS bound states of membranes and roots of the $A-D-E$ group. We derived a general bound on the light-cone energy of a membrane bound state and found that it had the necessary dependence on the blow-up parameters and longitudinal momentum. We also gave an explicit solution for all membrane bound states for the $A_{n-1}$ series. The 16-fold degeneracy of these solutions is consistent with the requirement that they form BPS vector multiplets in sevendimensional spacetime physics.

We were able to obtain all of the solutions that we expected from the M-Theory interpretation of the model, but we have not been able to show that we have given a unique solution. The existence of other solutions would probably lead to a greater than 16-fold degeneracy that would be hard to reconcile with the expected seven-dimensional physics. 
The solutions we found exhibit a very rich structure, and fit naturally into a noncommutative geometry framework. In section 4, we showed that there exist gauge-invariant coordinates for the membrane in the $A_{1}$ model that satisfy the relations appropriate to spherical membranes [18].

We then characterized the representations of excitations around the $A_{1}$ membrane in Section 5, finding that, as expected, only even spherical harmonics contribute in the multipole expansion. We then calculated the energy spectrum of the excitations and found the exact agreement with the expected result from the consideration of a large membrane, where we could trust both a calculation in a toy model, as well as in matrix model perturbation theory. The end result is very promising. The perturbation analysis gives the correct results in the large radius limit, and some coupling constants seem to decouple in the large $N$ limit. If this decoupling is a generic property of the large $N$ dynamics, this opens up the possibility of proving non-renormalization theorems.

Despite our success in solving for the membrane bound state solutions for the $A_{n-1}$ series, we have not been able to generalize them to the $D$ and $E$ series. This is basically due to the fact that our method of following the eigenvalues around the quiver diagram, while perfect for closed quivers, does not work for the $D$ and $E$ open quivers. By inspection of a few cases where $N$ is taken very small, one may also see that not all of the matrices in these cases can be diagonalized simultaneously. This is bound to complicate finding membrane solutions.

However, one should obtain similar results for the membrane properties in these cases. For example, the association of the BPS bound states with roots of the $A-D-E$ algebra and the bound on the energy of a bound state discussed in Section 3 are both completely general, so they apply equally well to the $D$ and $E$ series. Also, at large $N$, one expects that the couplings $g_{i} / g_{j}$ should still decouple from the low energy degrees of freedom (whose energies scale as $1 / N)$, as the states that feel these couplings should have energies of order 1 . Of course, even given a solution, in these cases the mass matrix is more complicated and should prove difficult to diagonalize.

There are still more tests that the matrix models for the ALE spaces should be put to. For example, it is particularly interesting to reproduce the Coulomb and velocity-dependent potentials between membranes and gravitons $[29,30,5,18]$. These and related matters are currently under investigation. 


\section{Acknowledgements}

We would like to thank Philip Candelas, Dan Kabat, and especially Willy Fischler for en-

lightening discussions about these and related issues. We thank Jacques Distler for his collaboration at an early stage of this work and many important conversations.

\section{References}

[1] T. Banks, W. Fischler, S. H. Shenker, and L. Susskind, "M Theory as a matrix model: A conjecture," Phys. Rev. D55 (1997) 5112-5128, hep-th/9610043.

[2] T. Banks, "Matrix theory," hep-th/9710231.

[3] D. Bigatti and L. Susskind, "Review of matrix theory," hep-th/9712072.

[4] W. Taylor, "Lectures on D-branes, gauge theory and M(atrices)," hep-th/9801182.

[5] D. Kabat and W. Taylor, "Linearized supergravity from matrix theory," hep-th/9712185.

[6] M. R. Douglas, H. Ooguri, and S. H. Shenker, "Issues in M(atrix) theory compactification," Phys. Lett. B402 (1997) 36-42, hep-th/9702203.

[7] M. Dine and A. Rajaraman, "Multigraviton scattering in the matrix model," hep-th/9710174.

[8] M. R. Douglas and H. Ooguri, "Why matrix theory is hard," hep-th/9710178.

[9] K. Becker and M. Becker, "On graviton scattering amplitudes in M theory," hep-th/9712238.

[10] S. Hellerman and J. Polchinski, "Compactification in the lightlike limit," hep-th/9711037.

[11] M. R. Douglas, "Enhanced gauge symmetry in M(atrix) theory," JHEP Elec. J. 7 (1997), no. 4, hep-th/9612126.

[12] W. Fischler and A. Rajaraman, "M(atrix) string theory on K3," Phys. Lett. B411 (1997) 53-58, hep-th/9704123.

[13] M. R. Douglas, "D branes and matrix theory in curved space," in Strings '97 (Amsterdam, June 16-21, 1997). hep-th/9707228.

[14] D.-E. Diaconescu and J. Gomis, "Duality in matrix theory and three-dimensional mirror symmetry," hep-th/9707019.

[15] D.-E. Diaconescu, M. R. Douglas, and J. Gomis, "Fractional branes and wrapped branes," hep-th/9712230. 
[16] D. Berenstein, R. Corrado, and J. Distler, "Aspects of ALE matrix models and twisted matrix strings," hep-th/9712049.

[17] B. de Wit, J. Hoppe, and H. Nicolai, "On the quantum mechanics of supermembranes," Nucl. Phys. B305 (1988) 545.

[18] D. Kabat and W. Taylor, "Spherical membranes in matrix theory," Adv. Theor. Math. Phys. 2 (1998), no. 1, hep-th/9711078.

[19] M. R. Douglas and G. Moore, "D-branes, quivers, and ALE instantons," hep-th/9603167.

[20] J. Polchinski, "Tensors from K3 orientifolds," Phys. Rev. D55 (1997) 6423-6428, hep-th/9606165.

[21] C. V. Johnson and R. C. Myers, "Aspects of type IIB theory on ALE spaces," Phys. Rev. D55 (1997) 6382-6393, hep-th/9610140.

[22] N. J. Hitchin, A. Karlhede, U. Lindstrom, and M. Rocek, "Hyperkähler metrics and supersymmetry," Commun. Math. Phys. 108 (1987) 535.

[23] P. B. Kronheimer, "The construction of ALE spaces as hyper-Kähler quotients," J. Diff. Geom. 19 (1989) 665-683.

[24] L. Susskind, "Another conjecture about M(atrix) theory," hep-th/9704080.

[25] M. B. Green, C. M. Hull, and P. K. Townsend, "D-brane Wess-Zumino actions, T-duality and the cosmological constant," Phys. Lett. B382 (1996) 65-72, hep-th/9604119.

[26] A. Connes, M. R. Douglas, and A. Schwarz, "Noncommutative geometry and matrix theory: Compactification on tori," hep-th/9711162.

[27] J. Polchinski, S. Chaudhuri, and C. V. Johnson, "Notes on D-branes," hep-th/9602052.

[28] J. Polchinski, "TASI Lectures on D-branes," hep-th/9611050.

[29] O. Aharony and M. Berkooz, "Membrane dynamics in M(atrix) theory," Nucl. Phys. B491 (1997) 184-200, hep-th/9611215.

[30] G. Lifschytz and S. D. Mathur, "Supersymmetry and membrane interactions in M(atrix) theory," Nucl. Phys. B507 (1997) 621-644, hep-th/9612087. 